\title{
El Proyecto Algebra: Un movimiento organizativo inspirado en Ella
}

\author{
Robert P. Moses, Mieko Kamii, \\ Susan McAllister y Jeffrey Howard
}

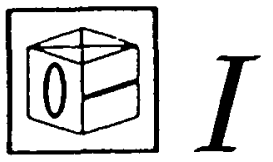

En este artículo se plantea uno de los problemas más centrales de la educación: el problema de la verdadera igualdad de oportunidades y del derecho de los individuos a planificar su curriculum. Aunque el sistema de selección estadounidense contra el que se rebelan los participantes en el proyecto Algebra pueda parecer muy lejano al sistema pretendidamente igualitario de nuestro país, hay un paralelismo entre ambos mayor de lo que pudiera pensarse. Los mecanismos de la selección son quizá menos explícitos en nuestro sistema curricular, pero igualmente efectivos y sus raices estriban en la separación entre la cultura escolar y la cultura familiar, entre el niño como alumno y el niño como ciudadano, entre los agentes educativos comunitarios $y$ los agentes educativos escolares.

Lo que los autores de este artículo plantean para el caso concreto del acceso al álgebra en las comunidades negras de Estados Unidos es igualmente válido para cualquier materia y para cualquier colectivo de individuos cuyo entorno social esté disgregado de la cultura oficial impuesta por la escuela: la necesidad de unir sentido con significado en el diseño de la educación. $\mathrm{O}$, lo que es lo mismo, plantear ésta como un desafio para el conjunto de la comunidad, recuperando para los nuevos contenidos que la sociedad exige los modos de enseñanza tradicionales, donde la motivación viene dada por la implicación de todos los agentes educativos de la comunidad en la instrucción del niño. 
Este artículo analiza el extraordinario impacto que ha tenido la organización de los derechos civiles -inspirada por Ella Bakeren los esfuerzos de Robert Moses, un padre activista de una comunidad. Moses, que también es matemático, sostiene que todos los niños deberían tener acceso al programa de estudios de preparación matemática universitaria de la escuela secundaria, y que a los niños que no tienen acceso a programas de este tipo se les está privando de los conocimientos y destrezas necesarias para participar en una economía que se rige por rápidos cambios tecnológicos. En este artículo, los autores describen la interacción entre padres, estudiantes y profesores implicados en El Proyecto Algebra: un esfuerzo de siete años para llegar a una pedagogía de las matemáticas cuyo objetivo es apoyar la generalización del estudio del álgebra en la escolaridad media*

El Proyecto Algebra, un programa dirigido a la enseñanza de matemáticas-ciencias en Cambridge, Massachusetts, ha organizado a las comunidades locales con el fin de ayudar a conseguir que el álgebra esté abierto a todos los estudiantes de séptimo y octavo curso, independientemente de su nivel previo de destreza o de rendimiento académico. La filosofía del proyecto es que el acceso a la enseñanza del álgebra permita que los estudiantes entren en los cursos de matemáticas y ciencias de nivel avanzado en la escuela secundaria, que a su vez constituyen la puerta de entrada para la universidad $\%$. El proyecto ofrece un nuevo plan

\footnotetext{
: En los Estados Unidos se está comenzando a plantear la enseñanza de las matemáticas en la etapa intermedia de la educación escolar. La Fundación Nacional de Ciencias (National Science Foundation, 1989), por ejemplo, ha publicado un escrito solicitando propuestas dirigidas a desarrollar material para la enseñanza de las matemáticas en esa ctapa en donde se exponen los elementos técnicos del problema con gran detalle. Pero en el núcleo de las cuestiones sobre la enseñanza de matemáticas-ciencias hay una pregunta política básica: Si la revolución tecnológica actual exige un nuevo nivel de habilidad en matemáticas y ciencias, ¿recibirán todos los ciudadanos el mismo acceso a las nuevas destrezas o se dejará a algunos atrás, negándoles una participación en la era económica y política que tenemos ante nosotros? Aquellos que se preocupen por las alternativas de vida existentes para las personas históricamente oprimidas en los Estados Unidos, no deben permitir que la educación de matemáticas-ciencias se aborde como si fuera puramente una cuestión de enseñanza técnica.

$\because$ En el sistema educativo de los Estados Unidos, a partir del grado 7 (12 años) comienza una selección de las asignaturas que dará el alumno - a partir de un programa base de estudios que varía enormemente según el Estado-con vistas a optar por uno de los distintos programas de estudio que comienza entre los grados 9 ó 10 y abarcan los 2 ó 3 últimos años de secundaria. A grandes rasgos, el alumno elige entre un programa dirigido a la enseñanza superior o un programa profesional
}

de estudios, y un proceso curricular para estudiantes de sexto curso compuesto por cinco pasos en el que se proporciona lo siguiente: una transición fluida desde los conceptos de aritmética a los de álgebra, aumentando la probabilidad del dominio del álgebra en séptimo y octavo; una cultura que abarque el hogar, la comunidad y la escuela en la que profesores, padres, miembros voluntarios de la comunidad, y administradores del centro escolar participen en actividades que fomenten el rendimiento académico de los estudiantes; $y$, un modelo de desarrollo intelectual basado en la motivación en vez de en la capacidad.

La actual creencia de que la capacidad es el principal ingrediente que guía el desarrollo intelectual y de que es necesaria para dominar las matemáticas avanzadas de la escuela secundaria es la base de la diferenciación existente en los planes de estudio de matemáticas de octavo curso, así como de la práctica extendida de ofrecer álgebra en octavo curso únicamente a aquellos estudiantes que están «matemáticamente predispuestos» o que tienen «talento» para las matemáticas. Los responsables del desarrollo del Proyecto Algebra han recurrido a la tradición del Movimiento para los Derechos Civiles para ayudar a las comunidades a organizar un desafío al modelo basado en la capacidad y sus manifestaciones institucionales.

\section{La Tradición del Movimiento para los Derechos Civiles en Mississippi}

La serie "La Mirada en el Premio" (Eyes on the Prize) de la PBS de los EE.UU. (Public Broadcasting System), ha brindado al público americano la oportunidad de recordar «la tradición de movilización de la comunidad" del Movimiento para los Derechos Civiles. Grandes masas de población se movilizaron para participar en acontecimientos a gran escala, como la campaña de Birmingham, la Marcha sobre Washington y la Marcha de Selma a Montgomery, cuyo objetivo era lograr que los negros del Sur tuvieran igualdad de oportunidades en el acceso a los medios e instituciones públicas.

(p. ej., agricultura, mercadotecnia, economía doméstica, mecánica u otros oficios). El programa académico está dirigido a preparar al alumno para el examen de acceso a la universidad. En este examen se evalúa principalmente la aptitud y la competencia verbal y matemática; la puntuación obtenida junto con el expediente escolar del alumno y las referencias de los profesores forman la base para que su solicitud sea o no aceprada. De ahí que, como explican los autores de este trabajo, los alumnos dirigidos a la universidad sean los únicos que reciben una preparación matemática completa. [N. de la T.] 
Personifica muy bien esta tradición el Dr. Martin Lutero King, Jr., quien dio vida al Movimiento inspirando a grandes masas de gente en enormes espacios públicos.

Dentro del Movimiento para los Derechos Civiles existía una «tradición para la organización de la comunidad" más antigua, aunque menos conocida. Esta tradición estableció los cimientos del "Verano para la Libertad del Mississippi» (Mississippi Freedom Summer, 1964) que revolucionó las relaciones interraciales en Mississippi, y la Ley del Derecho al Voto de 1965 (Voting Rigth $A c t$ ) que cambió la política de todo el Sur en el último cuarto de este siglo. Su lider era Ella Baker, una organizadora de la comunidad y fundi cuya sabiduría y asesoramiento guió a los negros veteranos de la primera oleada de protestas estudiantiles en el proceso de fundar y establecer el Comité de Coordinación No-violenta de Estudiantes (Student Nonviolent Coordination Committe $S N C C)^{1}$. Inculcó en los secretarios de campo del SNCC la creencia en la dignidad humana, la fe en la capacidad de los negros para generar líderes en las filas de su gente y la perseverancia al enfrentarse a enormes obstáculos. Baker simboliza la tradición del Movimiento para los Derechos Civiles de las zonas rurales y a los organizadores que llevaban estos lugares ${ }^{2}$. Así como su espíritu, su labor de concienciación y enseñanza, inspiraron al Movimiento del Mississippi, también impregnaron El Proyecto Algebra desde su comienzo.

Tres aspectos de la tradición organizativa del Movimiento de Mississippi subyacen en El Proyecto Algebra: el lugar central que ocupan las familias en el trabajo de organización; la delegación de la autoridad en la gente de base y su reclutamiento para el liderazgo; y el principio de "posa tu cubo donde estés", o la organización dentro del contexto en el que se vive y trabaja, trabajando en las cuestiones que surjen en este contexto ${ }^{3}$.

\section{Las familias y la organización}

La capacidad de las familias negras para adoptar, cuidar, querer y proteger a los organizadores de los Derechos Civiles como si fueran miembros de su familia tuvo una importancia central en el Movimiento de Mississippi. Esta práctica, conocida en la literatura como «absorción informal», permitió a los secretarios de campo y organizadores del SNCC y CORE (Congreso para la Igualdad Racial) trasladarse de un sitio a otro dentro de Mississippi prácticamente sin un dolar en el bolsillo, sabiendo que al final del viaje les esperaba la bienvenida de una familia. La absorción de los organizadores de los Derechos Civiles por familias negras era oro espiritual para el Movimiento de Mississippi, y daba a los organizadores del Movimiento la única credencial que nunca se podría ganar: ser uno de los hijos de la comunidad. Esta credencial contradecía la etiqueta de «agitador externo", utilizada en Mississippi por la estructura del poder Blanco para negar el impacto del Movimiento. Asimismo, los organizadores del Movimiento delegaban autoridad en sus familias adoptivas, reforzando las conexiones entre ellos y la amplia familia del movimiento que extendía sus redes por todo el país.

\section{La gente de base y el liderazgo de base}

El mensaje del Movimiento de Mississippi de conferir autoridad a la gente de base fue transmitido a todo el país a través de la televisión nacional durante la Convención $\mathrm{Na}$ cional Democrática de 1964 por aparceros, servicio doméstico y agricultores que formaban las filas del Partido Democrático para la Libertad en Mississippi (MFDP). Posteriormente, este mensaje fue llevado por organizadores de la comunidad Negros y Blancos a muchas áreas de actividad en la comunidad, incluyendo la educación, la sanidad, la asistencia social, la religión y la política. Pero ni el MFPD ni las otras organizaciones de base llegaron a arraigar ni desembocaron en un movimiento nacional fuerte de impulso de las personas negras. Las voces que se escucharon desde el Partido Democrático hasta el gobierno federal y desde el sector religioso hasta el sistema de enseñanza pública fueron las mismas: la idea de conferir un poder institucional a manos del "pueblo" negro es una noción demasiado problemática como para atraer un apoyo político duradero.

La idea del poder comunitario en la escuela pública, planteada por primera vez en Harlem en 1965 por organizadores de la comunidad negra, fue también respaldada por la América liberal Blanca. Por ejemplo, en 1969 se adoptó el Programa Abierto de la escuela Martin Lutero King, Jr., en las escuelas públicas de Cambridge, en parte debido a las reivindicaciones de los padres de Cambridge en pro de un mayor número de programas de Educación Abierta para sus hijos, y en parte debido a la demanda de supresión de la segregación racial en las escuelas de Cambridge ${ }^{4}$.

\section{“Posa tu cubo donde estés"}

Para dominar el arte de la organización de los movimientos de base, es necesario aprender 


\section{6}

a «posar tu cubo donde estés". En 1976, Bob y Janet Moses, ambos antiguos organizadores del SNCC en Mississippi, "posaron su cubo" en Cambridge y consideraron que el Programa Abierto del Colegio King era el lugar para educar a sus hijos ${ }^{5}$. En 1982, cuando Maisha, su hija mayor, comenzó octavo grado dentro del Programa Abierto, se puso en marcha lo que más tarde se convetiría en el Proyecto Algebra.

Antes de 1982, Moses, cuyo pasado incluía la enseñanza de matemáticas en secundaria en la ciudad de Nueva York y en Tanzania, había estado dando clases de matemáticas a sus hijos en casa. Maisha, ahora en su penúltimo año en la Universidad de Harvard, recuerda estas clases semanales durante el año escolar y diarias durante el verano y el resto de las vacaciones:

"Las matemáticas que dábamos en casa siempre fueron mucho más duras que las matemáticas del colegio. Era algo así como una tarea más de la casa. En nuestra familia, las clases extra de lectura con mi madre cuando éramos más pequeños y de matemáticas con mi padre eran parte de nuestra responsabilidad dentro de la familia, igual que lo era sacar la basura o hacer la colada».

Moses tuvo que enfrentarse a un reto familiar: la resistencia de los adolescentes a realizar lo que consideran una "pesada tarea doméstica». Maisha lo explica :

«Al ir creciendo, resultaba cada vez más duro lograr que nos pusiéramos a hacer matemáticas en casa. Nos enfrentábamos mucho más y nos quejábamos. "¿Por qué tenemos que hacer esto? Nadie más tiene que hacerlo". Papá solía decir, "Es importante. Quiero que lo hagais. Es necesario que lo hagais". Pero no nos quedabamos satisfechos. Yo no quería hacerlo. Papá se tenía que sentar ahí y luchar conmigo para que le diera las respuestas. Finalmemte decidió que la única forma de lograr que hiciera álgebra era introducirse en el colegio."

En el otoño de 1982, Mary Lou Mehrling, la profesora de octavo grado de Maisha, invitó a su clase a Moses para que diera álgebra a Maisha y a otros tres estudiantes de octavo. En la primavera, Maisha y otros dos estudiantes realizaron el examen de álgebra en la ciudad de Cambridge (Cambridge citywide algebra test) que se brinda a los estudiantes que desean superar el Algebra I y pasar directamente al curso de Algebra o Geometría avanzada en el noveno curso. Todos ellos aprobaron el examen, convirtiéndose en los primeros estudiantes de la historia del Colegio King en poder seguir el programa de estudios de matemáticas o ciencias avanzadas en la única escuela de secundaria en Cambridge: Cambridge Rindge and Latin ${ }^{6}$.
En parte pensando en su hijo mayor, que estaba a punto de comenzar séptimo grado del Programa Abierto, Moses decidió seguir trabajando durante el año siguiente (1983-84) con Mehrling y con otro profesor de séptimo/octavo grado. El número de estudiantes de octavo que daban álgebra con Moses aumentó a nueve. En el transcurso del año, los profesores seleccionaron a siete estudiantes de séptimo para dar álgebra el curso siguiente, creando el primer grupo de estudiantes de séptimo de «alta capacidad" que estarían bajo la dirección de Moses. En la primavera, los nueve estudiantes de octavo que Moses había preparado realizaron el examen de álgebra de la ciudad de Cambridge, aprobando seis de ellos.

$\mathrm{Al}$ año siguiente, el programa se amplió nuevamente, pero ya no era lo mismo. A partir de 1983-84 era evidente que, a pesar del compromiso de cubrir las necesidades educativas de todos los alumnos, la instrucción de matemáticas en el Programa Abierto estaba involuntariamente sesgada por factores raciales? Los alumnos de las dos clases de séptimo/octavo curso estaban agrupados de acuerdo con su "capacidad»: el nivel superior, compuesto principalmente por estudiantes blancos de clase media; el nivel inferior, compuesto casi exclusivamente por estudiantes negros y otros niños de color; y el nivel medio, donde había una mezcla racial. El sistema del Programa Abierto de segregar a los estudiantes en grupos según su capacidad, desviaba eficazmente a la mayoría de los estudiantes de color al carril en que no se daba álgebra, inculcando a demasiados jóvenes la noción auto-profetizada de que se espera poco de ellos.

Además, Moses y Mehrling se dieron cuenta de que algunos alumnos negros de alto rendimiento se sentían incómodos en el grupo de álgebra, porque esto suponía estar separados de sus amigos que se encontraban en los otros niveles de matemáticas. En general, la gente joven necesita identificarse lo más posible con sus iguales. La separación de los adolescentes académicamente dotados de sus iguales para hacerlos entrar en la "vía rápida» académica, puede agravar la ansiedad que acompaña el desarrollo de la identidad en la adolescencia ${ }^{8}$. Además la actitud hacia las matemáticas se modela a lo largo de la instrucción matemática en los niveles de séptimo y octavo. Tradicionalmente, en el sistema educativo de los Estados Unidos se introducen muy pocos principios matemáticos nuevos a lo largo de estos dos cursos y la atención se centra en el repaso (Usiskin, 1987). Moses y Mehrling hipotetizaron que si se utilizaran los cursos de séptimo 
y octavo para establecer una base de competencia en álgebra, podría aumentar la autoconfianza global de los estudiantes y proporcionarles los fundamentos matemáticos necesarios para acceder al nivel avanzado en secundaria.

La tradición organizativa del Movimiento de Mississippi se valía de los problemas cotidianos de la gente común, estructurándolos de manera que reportaran el máximo beneficio a la comunidad. En Mississippi, uno de esos problemas era el derecho al voto; técnicamente el planteamiento era: "¿Cuáles son los obstáculos jurídicos, legales, políticos y constitucionales para el derecho al voto? ¿Cómo podemos iniciar procedimientos judiciales sumarios, introducir legislación y movilizar apoyo político para retirar estos obstáculos?» Los trabajadores del SNCC y CORE persiguieron este objetivo situando a personas entre familias negras, por los condados más resistentes de todo el estado, que actuarían de base para luego seguir la expansión. Pero, los organizadores de Mississippi hicieron algo incluso de mayor importancia: concebir la cuestion del voto en su sentido político más amplio. En el transcurso de los esfuerzos por registrar a los votantes, los organizadores comenzaron a preguntarse a sí mismos y a la comunidad negra: «:Para qué es el voto? ¿En primer lugar, por qué lo queremos»? "Qué debemos hacer ahora mismo para asegurarnos de que cuando tengamos el derecho al voto servirá para beneficiar a nuestras comunidades?" Después de que los organizadores y los principales grupos de la comunidad hubieran estudiado una y otra vez estas y otras preguntas, cambiaron la estrategia de organización, hasta entonces dirigida al aumento del registro de votantes, y se centraron en sentar las bases para la creación de un partido político centrado en la comunidad, que se convirtió eventualmente en el Partido Democrático para la Libertad en Mississippi. La creacción de un nuevo partido político se convirtió en el objetivo principal del Movimiento de Mississippi debido a que tenía mayor potencial para implicar a las personas de la comunidad en un esfuerzo sustancial a largo plazo. Los participantes llegarían a considerar las preguntas políticas y sus respuestas como suyas propias".

En el Programa Abierto el problema que se planteaba era la enseñanza de álgebra en séprimo y octavo. Moses, el padre-como-organizador del programa, utilizó instintivamente las lecciones que había aprendido en Mississippi, transformando los problemas cotidianos en una pregunta política más amplia para la comunidad del Programa Abierto: «¿Para qué sirve el álgebra? ¿Por qué queremos que los niños lo estudien? ¿Qué necesitamos incluir en la educación matemática de cada estudiante en la etapa intermedia de la educación escolar para que cada uno tenga acceso al programa de estudios de preparación matemática universitaria en la escuela secundaria? ¿Por qué es importante tener este acceso?

Mediante la asociación del contenido de la educación matemática con las perspectivas futuras de los estudiantes de áreas urbanas, Moses transformó lo que hasta entonces había sido una cuestión puramente curricular en una cuestión política más amplia. Utilizando su experiencia como organizador, educador y padre, Moses transformó el diálogo entre padres, profesores y administradores escolares del Programa Abierto en un diálogo centrado en preguntas que llegasen al corazón de la práctica educativa: ¿Cómo se puede crear en el Programa Abierto una cultura dirigida a que todos los niños alcancen el mejor nivel posible en su desarrollo matemático? ¿Cuál debe ser el contenido de las matemáticas que se dan en la etapa intermedia escolar? ¿Cuáles son los procesos curriculares que hacen que ese contenido este abierto a todos los estudiantes?

La piedra angular en el desarrollo del Proyecto Algebra fue por tanto la expectativa de que cada niño en el Programa podría alcanzar destreza en matemáticas; un principio suficientemente poderoso como para que se extendiera tanto por la cultura de los estudiantes como por la de los adultos. Los componentes de este esfuerzo incluían cambiar el contenido y los métodos de enseñanza de las matemáticas, la participación de los padres en actividades que les permitiesen apoyar mejor el aprendizaje de sus hijos, enseñar a los estudiantes a establecer metas y motivarles para alcanzarlas, y contactar a licenciados negros en el área de Boston para que actuasen como tutores y modelos del rol de éxito académico.

\section{Los profesores como estudiantes}

Desde el principio, Moses y Mehrling modelaron la noción de que no es deshonra admitir ignorancia si éste es el primer paso para aprender. Mehrling, una antigua profesora de música, comenzó a estudiar matemáticas, empezando con álgebra y eventualmente obteniendo el certificado estatal de matemáticas. Pero hizo algo más profundo: convirtió su falta de experiencia en matemáticas en un componente de aprendizaje al adoptar una postura de inquisición mútua con los estudiantes y presentándose como una persona que estaba aprendiendo. Como ella misma afirma, «desarrollé 
formas de responder a las preguntas de los estudiantes que les ayudase tanto a ellos como a mí misma a pensar a fondo el problema hasta el final». Cuando le surgían preguntas, se las planteaba al momento a Moses.

«Presentarme ante mis alumnos como alguien que está aprendiendo me ayudó a comprender lo que estaban experimentando y a ellos les ayudó a sentirse más tranquilos cuando pedían ayuda. Los estudiantes dejaron de sentirse amenazados cuando no comprendían un problema o un concepto, pues vieron que todos estábamos aprendiendo y que todos aprendemos de manera diferente».

Como Mehrling se presentó abierta y honestamente como estudiante de la asignatura que enseñaba, pudo aumentar la confianza de sus alumnos. Abiertamente transmitía el mensaje: «Si yo [vuestra profesora] puedo arriesgarme a quedar en evidencia para aprender esta asignatura, seguro que vosotros también podéis hacerlo». Pero además les transmitía un poderoso mensaje latente:

"Estoy convencida de que las personas que no conocen esta asignatura puedan apenderla; para ello tienen, en todo momento, que estar dispuestos a no aparentar que comprenden lo que realmente no comprenden; para aprenderla no debe importarles pedir ayuda y deben estar dispuestos a arriesgarse a pasar vergüenza».

El mensaje de Mehrling recapitulaba un mensaje memorable que Fannie Lou Hamer y otros habían transmitido en el punto culminante del desafío lanzado por el MFPD a la Convención Democrática Nacional de 1964: la confianza de que las personas que no conocían el tema de la política podían aprenderlo haciendo preguntas directas y arriesgándose a pasar verguenza. Cada persona se enfrentó a su falta de experiencia con honestidad e integridad, transformando las desventajas latentes en elementos de fuerza.

\section{La participación de los padres}

Desde su comienzo, el Programa Abierto había desarrollado una política y práctica que fomentaban la participación activa de los padres en la contratación de personal, en el desarrollo de programas de estudio, en la observación y evaluación del profesorado y en la dirección y administración del colegio. En este contexto, la participación de los padres en el Proyecto Algebra fue creciendo de forma natural.

Los padres que en 1984-85 formaron parte del comité del Programa de séptimo/octavo curso concluyeron que la decisión de estudiar álgebra en los cursos séptimo y octavo no podía dejarse exclusivamente a los alumnos de sexto curso. Eran demasiado jóvenes para comprender totalmente las implicaciones a largo plazo de esta decisión a la hora de entrar en la universidad. Esta decisión tampoco debería recaer exclusivamente en el profesorado, en los coordiandores del plan de estudios, o en los administradores del colegio o del área escolar, cada uno de los cuales tenía sus propias ideas sobre quién debería estudiar álgebra y en qué curso. Más bien era necesario que los padres participaran en la toma de decisiones de carácter educativo sobre sus hijos, tanto a nivel individual como para establecer una política escolar. También era necesario que estuviesen mejor informados sobre los aspectos concretos del plan de estudios de matemáticas de la etapa intermedia escolar, con el fin de que pudieran tomar decisiones con conocimiento del tema y proteger los intereses de sus hijos.

Durante la primavera de 1985, un padre en el comité del Programa Abierto de séptimo/octavo curso colaboró con Moses en la distribución de una carta dirigida a los padres de todos los estudiantes de sexto, preguntándoles si consideraban que todos los alumnos de séptimo curso deberían estudiar álgebra y si en el caso específico de su hijo pensaban que éste debería dar álgebra en séptimo curso. En respuesta, algunos padres opinaron que probablemente había estudiantes de séptimo que no estaban preparados, pero ninguno consideró que a su hijo se le debería negar acceso al álgebra en séptimo curso. Al exponer las contradicciones entre las evaluaciones de los padres sobre las aptitudes de sus hijos y los supuestos de los planes de estudio de la comunidad se logró crear un consenso en torno al resultado educativo de todos los chicos.

Este fue el catalizador para invitar a todos los niños dentro del Programa Abierto, comenzando séptimo en el otoño de 1985, a estudiar álgebra tres veces por semana. Con la excepción de algunos estudiantes de octavo curso que en la opinión de sus profesores «no estaban listos», la invitación de estudiar álgebra también se extendió a todo octavo. El consenso de los padres puso en marcha un cambio en la política y cultura del colegio. Actualmente, se asume que cada estudiante del Programa Abierto estudiará álgebra en séptimo y octavo.

$\mathrm{Al}$ ir avanzando el Proyecto, la participación de los padres aumentó, ofreciéndose como voluntarios en las clases y participando en talleres sobre la autoestima y el rendimiento de los estudiantes. Se invitó a todos los padres del Colegio King a asistir a las reuniones del grupo 
de padres "Honors Bound", que preparaba a los estudiantes de color para el reto que suponía optar por los cursos de nivel avanzado en secundaria, y que creó una cultura hogar-colegio dirigida a estimular y apoyar el esfuerzo intelectual serio. Se puso en marcha un cursillo de álgebra para los padres los sabados por la mañana, en el que el álgebra se enseñaba de la misma manera que a sus hijos.

Los padres que asistían a estas clases de álgebra de los sábados se comprometían a hacer el Proyecto "suyo" en un sentido fundamental. Un padre agradecido captó las múltiples dimensiones de esta experiencia en una carta al Comité Escolar de Cambridge en 1987:

«...para mí este programa ejemplifica mis máximos deseos para la educación de mi hija y el resto de la gente joven de nuestra comunidad: una orientación positiva hacia el aprendizaje; una rica comprensión de las matemáticas avanzadas; el reconocimiento de la relación entre lo que se aprende en clase $y$ lo que sucede en la vida; y una sensación de autoridad personal

Como estudiante de sexto en su primer año en el programa, mi hija comenzó a superar el miedo hacia las matemáticas y la percepción distorsionada de lo que es capaz de hacer y de por qué algo es importante. Creo que esto se debió a diversos factores, la sensación de que tanto estudiantes, como profesores y ayudantes estaban aprendiendo juntos; la desmitificación de la asignatura al relacionarla con experiencias reales; y el hecho de que su madre, junto con otros padres y miembros de la comunidad, estaban simultáneamente superando un pánico latente hacia las matemáticas en el cursillo de los síbados.

Esta e xperiencia no solamente me ayudó a comprender el programa (y a aprender matemáticas); también me aumentó en gran medida mi comprensión de la vida en el colegio y en la comunidad local, y de los problemas que como ciudadano puedo ayudar a resolver..."

Los padres recibieron numerosas cartas, ofreciéndoles la oportunidad de hablar, hacer preguntas, y participar en la planificación del proyecto. Todo ello como reconocimiento del papel central de los padres en la construcción de una cultura hogar-colegio de alto rendimiento.

\section{La creación de un nuevo entorno de enseñanza y aprendizaje para las matemáticas}

Como factor adjunto al hecho de poner el álgebra a disposición de todos los alumnos de séptimo y octavo en 1985 , el sistema de agrupamiento según la capacidad del estudiante fue sustituído por la enseñanza individualizada y en pequeños grupos. Se enseñaron destrezas de aprendizaje a los estudiantes para que aprendieran el material fuerte "por sí solos". En reuniones con los profesores, se pidió a los estudiantes que establecieran sus propios objetivos a corto plazo (por ejemplo, decidir el número de lecciones que querían completar cada semana), y metas a más largo plazo (por ejemplo, tomar la decisión de prepararse para el examen de la ciudad de Cambridge). Los padres eran informados de estas metas y se les pedía que cada semestre firmaran un informe en el que constaban las metas de sus hijos.. El ritmo y la extensión de los estudios de matemáticas estaban, por tanto, bajo el control del estudiante. Mehrling relata una historia que refleja la motivación individual y grupal que un sistema de establecimiento de metas de este tipo puede fomentar:
"Andrea habló en voz alta en una de nuestras primeras reuniones y dijo, "Voy a hacer cuatro lecciones a la semana porque quiero terminar tal y tal para el final de séptimo grado, para así poder terminar el libro para el final de octavo, y así puedo entrar en la clase de geometría avanzada en noveno grado". Esto lo dijo una alumna de doce años. Los demás la miraron - esto no había venido del profesor-y dijeron, "Estás loca?" Ella dijo, "Eso es lo que voy a hacer". Bob [Moses] estaba ahí y comenzó a explicarles por qué lo que Andrea acababa de hacer era un acto muy maduro y con visiones a largo plazo, y por qué quizás ellos no estaban todavía preparados para hacer lo mismo. Esto dio a Andrea mucho apoyo y afirmación por haber dicho eso en el grupo. Y cambió lo que los demás iban a decir. Todo a partir de entonces era en relación a Andrea: "Bueno no voy a hacer exactamente lo mismo que Andrea, pero..." "

Asimismo, los estudiantes aprendieron a trabajar más duro de lo que lo habían hecho anteriormente. Se fomentó el desarrollo de hábitos de concentración, paciencia y perseverancia al enfrentarse con su trabajo diario de matemáticas. Los estudiantes decidían cuál de diversas fuentes debían consultar -el libro de texto, el instructor, un compañero- cuando tenían una pregunta o dificultad para resolver un problema de matemáticas. Los profesores se reunían con los alumnos en pequeños grupos impartiendo clases cortas sobre conceptos específicos, y regularmente mantenían sesiones de repaso en pequeños grupos. Pensando en esta decisión Mehrling explicó recientemente:

"Los estudiantes adolescentes pueden a veces interrelacionarse con el material y esto no resulta tan amenazante como la interacción con un adulto. Si pueden ir a un adulto a preguntarle sobre el material cuando están listos para recurrir al adulto, es totalmente distinto a estar en grupo, ser señalado y puesto en evidencia; sintiéndose vulnerable sobre 
las partes que todavía no han encajado en su mente. Una vez que comienzan a interactuar con el material, no solamente se vuclven muy posesivos del mismo, sino también muy reacios a volver a cualquier tipo de clases dirigidas por profesores. De una forma curiosa, el material les da autoriclad -algo que nunca se me hubiera ocurrido-. Generalmente, el Programa Abicrto es un tipo de programa con una fuerte direción por parte del profesorado. Somos los encargados de motivar a los alummos, traer el material de todos los sitios, y nuestra enseñanza es interpersonal. Descubrimos en los niveles de séptimo y octavo que ese era uno de los problemas con los estudiantes que se sentían vulnerables: les ponía en situaciones incómodas."

Como parte del nuevo entorno curricular, pedagógico y social para el estudio de las matemáticas, el profesorado de los cursos séptimo y octavo en su relación con los estudiantes asumió el papel de "preparador" en contraposición al de "persona que imparte clases».

La primera clase completa de estudiantes del Proyecto se graduó en la primavera de 1986. Cuando el siguiente otoño comenzaron la escuela secundaria, el 39 por ciento entró en Geometría o Algebra Avanzada. No hubo un sólo estudiante de esa cohorte que terminara en el nivel inferior de las clases de matemáticas, como el Algebra I, del Colegio Cambridge Rindge y Latin.

\section{Ampliación del plan de estudios}

En 1986 la atención se volcó en preparar a los estudiantes para el álgebra de séptimo curso. Ahora que todos los estudiantes de séptimo y octavo daban álgebra, los profesores de los cursos inferiores comenzaron a cuestionar la adecuación de sus programas de estudio de matemáticas como preparación para el álgebra. Para abordar esta cuestión de forma sistemática, la totalidad del personal del Programa Abierto participó en un seminario de un año de duración centrado en la cuestión de la destreza matemática.

Al finalizar el seminario, los profesores de todos los niveles (desde preescolar a octavo) pusieron en marcha los nuevos programas de estudio de matemáticas, cada uno apropiado para la edad y el nivel en que enseñaban. A algunos profesores les preocupaba tener que diseñar sus propias prácticas curriculares en torno a las necesidades de los niños y su estilo de enseñanza. Los resultados del Proyecto Algebra sugieren que la flexibilidad lleva a una mejor pedagogía. Por ejemplo, el profesorado de quinto/sexto intentó establecer un enfoque centrado en el material para los estudiantes de sexto que había dado muy buenos resultados en los niveles de séptimo y octavo; pero encontraron que los niños más jóvenes, acostumbrados a una instrucción dirigida por el profesor, necesitaban que en el programa de estudios de transición de sexto curso hubiera mayor interacción profesor-alumno y en pequeños grupos. Los profesores modificaron sus técnicas en el aula, pero mantuvieron el principio de fomentar mayor autonomía para hallar las respuestas a los problemas. La mejor adaptación del programa de estudios fue en sí algo beneficioso. Pero también de igual importancia, este proceso dio a los profesores la misma sensación de poder experimentado por los estudiantes. Era más probable que los profesores que participaron en las innovaciones del programa de estudios y en el entrenamiento propio sobre cómo presentarlo, comprendiesen, apreciaran y fomentaran la destreza de auto-educación que era un concepto contral para el Proyecto Algebra. Un profesor explicó:

«Bob apoyaba lo que hacíamos mientras nos ayudaba a cambiar. Nunca entró y dijo, "Vamos a tirar esto, no sirve". Sino que decía, "Sois maravillosos. ¿Queréis probar algo diferente?" Cuando preguntamos " ¿Cómo funcionará?" le daba vuelta y respondía, "Bueno, cómo creéis que debería funcionar? ¿Qué es lo que queréis que suceda?" No nos dio realmente una forma, que admito resultó frustrante, pero esto también nos concedió un sentido de posesión en torno a ello. Bob no tenía todas las respuestas. Al principio me sentía realmente enfadada de que me estuviera haciendo pasar por todo este proceso. No dejaba de decir "Bob tiene un calendario. ¿Por qué no nos lo cuenta? ¡Estamos perdiendo un montón de tiempo!" Pero él sabía que tenía que salir de nosotros. Sabía que no podía imponerse, porque no sabía qué funcionaría. No era profesor de un curso. Simplemente tenía una visión. Si podía ayudarnos a captar esa visión, nosotros lo haríamos funcionar.»

Un segundo resultado fue que Moses acordó desarrollar un programa de estudios para los alumnos de sexto que proporcionase una transición conceptual desde la aritmética al álgebra. Los aspectos principales de lo que se ha dado a conocer como el Proyecto Algebra y la filosofía que guió su elaboración se discuten a continuación.

\section{Qué enseñar y cómo hacerlo}

$\mathrm{Al}$ abrirse las puertas del álgebra a todos los estudiantes en 1985-86, Moses tuvo la oportunidad de trabajar de cerca con varios estudiantes que tenían grandes dificultades con los primeros capítulos del libro de texto de álgebra. En particular había un alumno negro que tardó muchos meses en completar las primeras lec- 
ciones. Moses se preguntaba dónde exactamente estaría el nudo conceptual del estudiante. ¿Era posible guiar al estudiante desde la aritmética al álgebra elaborando un mapa conceptual que comenzase con conceptos que eran obvios y procediera a través de pasos igualmente obvios?

Después de haber trabajado con un número de estudiantes que estaban teniendo dificultades, Moses llegó a la conclusión de que el centro del problema estaba en el concepto de número de estos alumnos. En aritmética, el rasgo distintivo de un número es la magnitud y la cantidad. En álgebra el número tiene dos rasgos distintivos: uno es cuantitativo; el otro es cualitativo, y debe ser enseñado explícitamente. Los estudiantes de aritmética tienen en sus cabezas una pregunta que asocian con contar números: «¿Cuántos?» Los estudiantes de álgebra necesitan tener dos preguntas: «¿Cuántos?», y otra más del tipo de «¿En qué dirección?" como puntos de referencia para el concepto intuitivo de los opuestos. Los niños comprenden la pregunta "¿En qué dirección?» desde sus primeros años, pero no es algo que asocien con el número. El concepto de número utilizado en la aritmética debe generalizarse en el álgebra, y si esta generalización no sucede se bloquea la comprensión de los estudiantes. Una vez que los estudiantes han generalizado su concepto de número, también deben generalizar su conocimiento de operaciones básicas como la resta.

Gradualmente Moses elaboró un proceso de enseñanza-aprendizaje compuesto por cinco puntos que lleva al estudiante desde el suceso físico a su representación simbólica, de esta forma se acelera la comprensión de los estudiantes de sexto de los conceptos clave necesarios en el estudio de álgebra ${ }^{10}$. Los cinco pasos son:

\section{Suceso físico}

2. Dibujo o modelo de este suceso

3. Descripción linguística intuitiva (ideomática) de este suceso

4. Una descripción de este suceso en lenguaje reglamentado

5. Representación simbólica del suceso

El objetivo de los cinco pasos es evitar la frustración del estudiante en «el juego de las señales", o la idea equivocada de que las matemáticas son la manipulación de una colección de símbolos y signos misteriosos. Chad, un joven estudiante negro de séptimo curso, recientemente, tras haber leído una página del primer capítulo de un texto tradicional de álgebra levantó la vista y le dijo a su madre, «son sólo un montón de palabras». Para demasiados jóvenes las matemáticas son un juego de signos al que no pueden jugar. Es necesario ayudarles a comprender qué es lo que esos signos realmente significan, y construir para sí mismos una base de pruebas a favor de las matemáticas. Cuando los estudiantes de la etapa intermedia escolar utilizan el proceso de cinco pasos para construir la representación simbólica del suceso físico (representaciones que ellos mismos se inventan), los estudiantes forjan, a través de la experiencia directa, su propia plataforma de verdades matemáticas. Las representaciones simbólicas que ellos mismos han construido entran dentro de un sistema de verdades matemáticas que poseen contenido y significado.

En el Programa Abierto, los estudiantes inician este proceso con una excursión por la Línea Roja del sistema de metro de Boston (el suceso físico). Esta experiencia proporciona el contexto en el que se pueden hacer un número de preguntas obvias: ¿En qué estación comenzamos? ¿A dónde vamos? ¿Cuántas paradas habrá hasta la llegada? ¿En qué dirección vamos? Estas preguntas tienen respuestas obvias y forman la base de las matemáticas de la excursión. Cuando vuelven, se pide a los estudiantes que escriban sobre la excursión, que dibujen un mural o construyan un modelo tridimensional, que realicen gráficos de excursiones que ellos planifican y que recojan datos estadísticos sobre ello. El objetivo es fusionar en sus mentes las dos preguntas: «¿Cuántos?» y «¿En qué dirección?" y anclar estas preguntas a sucesos físicos.

Los estudiantes entonces utilizan este concepto para explorar el concepto de equivalencia en el amplio contexto cultural de los acontecimientos cotidianos como, por ejemplo, cocinar, entrenar, enseñar, pintar y hacer reparaciones. Exploran cualquier concepto en el que un objeto $A$ es sustituido por otro objeto B para alcanzar un objetivo específico. Concluyen la discusión de equivalencias de la excursión por el metro con construcciones abiertas de excursiones equivalentes, dirigidas hacia una introducción sobre los desplazamientos, como «excursiones que tienen el mismo número de paradas y van en la misma dirección".

Una vez que se han introducido los desplazamientos, los alumnos investigan el concepto de "comparar" como paso anterior a la generalización del concepto de resta. La mayoría de los textos de álgebra introducen la resta como un problema de adición transformado. Se pide a los estudiantes que piensen en la resta $(3-(-2)=+5)$ como «sumar el opuesto" 0 "averiguar el sumando que falta» $(3-$ ? 
$=5$ ), que proporciona un grupo de signos que sirve de referencia para el otro. Pero, los estudiantes buscan experiencias concretas, dibujos, o al menos un concepto, para asociar directamente con la resta algebráica. El problema se complica porque los estudiantes han sobreaprendido el concepto "quitar de" como algo suyacente a la resta. En álgebra, "quitar de» ya no tiene una aplicación directa a la resta. A los pocos meses de comenzar a dar álgebra, los estudiantes se enfrentan a enunciados de resta sin un contenido discernible, sólo tienen un signigicado indirecto relacionado con un problema asociado de adición y no son en absoluto evidentes.

Para dar mayor significado, contenido y claridad a la resta, al comenzar álgebra, los estudiantes empiezan con el evento físico de comparar la altura de dos estudiantes, Costacosta que tiene una altura de $180 \mathrm{~cm}$ y Miramé, que mide $120 \mathrm{~cm}$. La clase trabaja con un dibujo de este suceso, generando preguntas que se pueden utilizar para comparar la altura:

1. ¿Cuál es más alto?

2. ¿Cuál es la diferencia de altura?

3. ¿Cuánto mide Miramé menos que Costacosta?

4. ¿Quién mide menos?

5. ¿Cuánto mide Costacosta más que Miramé?

En aritmética hay dos conceptos de resta, el concepto de «quitar de» y el concepto de «la diferencia entre». Este último proporciona la entrada apropiada para la resta en álgebra, como se ilustra en las preguntas que se presentaron anteriormente. Los estudiantes identificarán fácilmente la respuesta a la segunda pregunta utilizando la resta para averiguar la diferencia en altura. Esto les prepara para acep- tar la resta como la mejor forma de abordar las respuests de tipo comparativo; preguntas que pertenecen al álgebra y no a la aritmética.

Las respuestas a estas preguntas se procesan cuidadosamente en tres etapas: lenguaje intuitivo, lenguaje reglamentado, y representaciones simbólicas. ¿Cuánto mide Costacosta más que Miramé? se explora de la siguiente manera:

- Lenguaje intuitivo: "Costacosta es $60 \mathrm{~cm}$ más alto que Miramé

- Lenguaje reglamentado: «La altura de Costacosta comparada con la altura de Miramé es $60 \mathrm{~cm}$ mayor".

- Representaciones simbólicas:

(5a) A (C) comparada con A (M) es $60 \mathrm{~cm} 1$ $A(C)-A(M)=60 \mathrm{~cm}$

(es decir, $180 \mathrm{~cm}$ es $60 \mathrm{~cm}$ más alto que 120 $\mathrm{cm}$ )

“¿Cuánto mide Miramé menos que Costacosta?» procede por un camino similar.

- Lenguaje intuitivo: "Miramé mide $60 \mathrm{~cm}$ menos que Costacosta».

- Lenguaje Reglamentado: «La altura de Miramé comparada con la altura de Costacosta es $60 \mathrm{~cm}$ menor».

- Representaciones simbólicas:

(3a) A (M) comparada con A (C) es $60 \mathrm{~cm}$ !

$$
\mathrm{A}(\mathrm{M})-\mathrm{A}(\mathrm{C})=60 \mathrm{~cm} \text { ! }
$$

(es decir, una altura de $120 \mathrm{~cm}$ es $60 \mathrm{~cm}$ menor que una altura de $180 \mathrm{~cm}$ )

Esta forma de comparar cantidades físicas se refuerza fácilmente con estaciones de trabajo en donde los estudiantes comparan pesos, longitudes, temperaturas y velocidades. Pueden volver a su experiencia en el metro para comparar las posiciones de las estaciones de la Línea Roja urilizando el siguiente modelo.

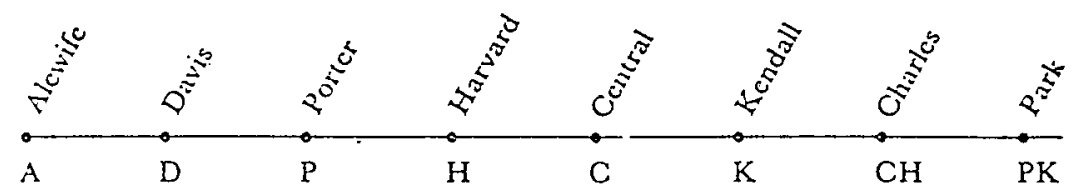

Cuando se pregunta, “Cuál es la posición de Harvard en relación con la de Kendall?» los estudiantes realizan los siguientes pasos:

- Lenguaje intuitivo: «Harvard está a dos paradas al oeste de Kendall».

- Lenguaje reglamentado: «La posición de Harvard comparada con la posición de Kendall es de dos paradas hacia el oeste».
- Representaciones simbólicas:

(a) $\mathrm{P}(\mathrm{H})$ comparada con $\mathrm{P}(\mathrm{K})$ es $\overleftarrow{2}$

(b)

$\mathrm{P}(\mathrm{H})-\mathrm{P}(\mathrm{K})=2$

De forma similar, la pregunta «¿Cuál es la posición de Kendall en relación con la de $\mathrm{Har}$ vard?" produce:

$\mathrm{P}(\mathrm{K})-\mathrm{P}(\mathrm{H})=\overrightarrow{2}$ 
Tan pronto como se introducen los números enteros como sistema de coordenadas, los estudiantes están listos para generar sus propios problemas de resta. Al haber introducido anteriormente la noción de un punto arbitrario de refërencia, se asignan a las estaciones sistemas de coordenadas colocando el punto del cero alternativamente en distintas estaciones. Cada asignación genera un problema de resta diferente para la pregunta, " $i C u a ́ l$ es la posición de Harvard en relación con la de Kendall?»

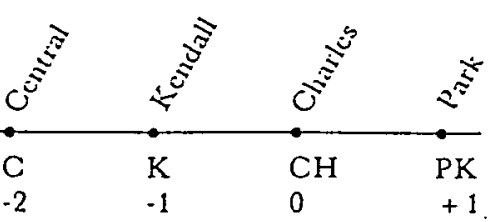

(a) $\mathrm{P}(\mathrm{H})$ comparada con $\mathrm{P}(\mathrm{K})$ es $\overline{2}$

(b) $\quad \mathrm{P}(\mathrm{H})-\mathrm{P}(\mathrm{K})=\overleftarrow{2}$

(c) $\quad-3-(-1)=-2$

Siguiendo el mismo razonamiento, la pregunta «¿Cuál es la posición de Kendall en relación con la de Harvard?» produce:

$$
\begin{aligned}
& \mathrm{P}(\mathrm{K})-\mathrm{P}(\mathrm{H})=2 \\
& -1-(-3)=+2
\end{aligned}
$$

Las comparaciones opuestas $[\mathrm{P}(\mathrm{H})$ comparado con $\mathrm{P}(\mathrm{K})$, y $\mathrm{P}(\mathrm{K})$ comparado con $\mathrm{P}(\mathrm{H})$ ] llevan a expresiones opuestas $[(-3)-(-1)$, $\mathrm{y}(-1)-(-3)]$ y a números enteros opuestos $[(-2)$ y $(+2)]$, de una manera que da un significado directo e intuitivo a la resta de números enteros y otorga a estudiantes y profesores por igual el control sobre la generación de problemas y ecuaciones de resta sencillas. El currículo y proceso curricular utilizado en sexto curso han hecho que el álgebra sea accesible a todos los estudiantes de la etapa intermedia escolar. El Proyecto ha demostrado que todos los estudiantes de séptimo y octavo en el Programa Abierto del Colegio King pueden estudiar álgebra, y que la totalidad de la comunidad escolar espera que así lo hagan.

\section{Participación comunitaria en la creación de una cultura dirigida al rendimiento académico}

Para los jóvenes que se han sentido excluídos de la cultura del éxito académico en el colegio, la expectativa de que ellos también pueden aprender es crucial. Durante el curso escolar 1987-88, la respuesta del Proyecto a los chicos que pensaban que no tendrían éxito en matemáticas fue institucionalizar una serie de medidas diseñadas a crear una cultura de destreza matemática y científica, no sólo en el Programa Abierto sino también en otros programas de estudio del Colegio King. El Instituto Seymour para Estudios Cristianos Avanzados (Seymour Institute for Advanced Christian Studies), una organización de servicio concebida por licenciados negros de Harvard para apoyar el desarrollo con base en la comunidad de áreas urbanas, proporcionaba modelos de rol negros para entrar en las aulas y dar clases de apoyo a los estudiantes, $y$, además, organizaban aulas de estudio de álgebra cuatro días por semana antes de que comenzara el colegio. Las aulas de estudio estaban abiertas a estudiantes de séptimo y octavo pertenecientes a los cuatro programas educativos existentes en el Colegio King. Los tutores, procedentes de Harvard, MIT, el Instituto Wentworth y la Universidad de Boston, establecieron relaciones con los niños a nivel individual y para los estudiantes de séptimo y octavo se convirtieron en modelos del rol de adultos jóvenes que habían alcanzado el éxito académico. Un estudiante de Derecho de Harvard y tutor escribió:

"me ha impresionado el hecho de que estos estudiantes de séptimo y octavo curso sean capaces de leer y comprender sus libros de texto de matemáticas; poseen cierta comprensión de los conceptos algebraicos y están dispuestos a venir a las 7:30 de la mañana para trabajar en sus habilidades matemáticas... Los estudiantes en el Proyecto Algebra son capaces de ayudarse a sí mismos y unos a otros utilizando sus libros. En el Proyecto ayudarse mutuamente tiene otro papel importante. Creo que son las amistades lo que hace que sigan viniendo a las aulas de estudio a primera hora de la mañana; se están estableciendo fuera de las clases relaciones que apoyan el rendimiento académico."

Al irse desarrollando el Proyecto Algebra, el mensaje de que todos los niños podían aprender fue articulado de forma más sistemática por el Instituto Efficacy ${ }^{11}$. El modelo "Efficacy" ofrece a educadores, padres y estudiantes una alternativa explícita al modelo de aprendizaje basado en la capacidad, al subrayar la confianza y los esfuerzos eficaces como ingredientes clave en el proceso de desarrollo intelectual. "Efficacy" 
asume que los niños, que están lo suficientemente dotados como para dominar los fundamentos del lenguaje a una edad temprana, son totalmente capaces de aprender matemáticas. Para aprender es necesario que los niños canalicen su esfuerzo de manera eficaz. Deben aprender a trabajar con dedicación, concentración y estrategias fiables. Cuando el aprendizaje se percibe en función de un esfuerzo efectivo, es necesario buscar los factores que están inhibiendo a los chicos cuando tienen dificultades en el aprendizaje o en la comprensión de un concepto, en vez de buscar «incapacidades» que sirvan para rechazar el aprendizaje.

Muchos niños de color aprenden desde una temprana edad que existen dudas sobre su capacidad de desarrollo intelectual. Mensajes transmitidos en el medio escolar (los estudiantes son puestos en grupos de capacidad inferior), en el grupo de compañeros (un anti-intelectualismo contagioso dentro del grupo de compañeros), y a través de los medios de comunicación (expectativas de inferioridad), contribuyen, todos ellos, a inculcarles que puede que no estén a la altura de la tarea de realizar estudios avanzados. La falta de confianza engendrada por la internalización de estos mensajes modela el significado de cualquier fracaso («Supongo que esto prueba que no soy listo») y va minando la capacidad para trabajar ( ¿Para qué me voy a estar golpeando la cabeza contra la pared si de todas formas soy incapaz de aprenderlo?").

Para cambiar estas circunstancias, el modelo «Efficacy» trabaja para implantar una idea alternativa en la mente del niño: «Si trabajo lo suficientemente duro, puedo ser más listo que nadie»:

\section{Confianza (Piensa que puedes)

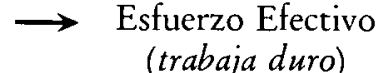 \\ (trabaja duro)

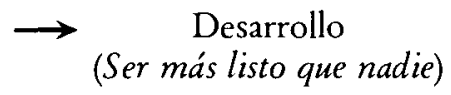

El énfasis se pone en el proceso de desarrollo, y cierta medida de control se devuelve al niño.

Los profesores son los portadores de las ideas del modelo "Efficacy», y en ellos recae la responsabilidad de construir la confianza de los alumnos y de modelar el esfuerzo firme. Los profesores asisten a un seminario intensivo de cinco días de duración para aprender el modelo «Efficacy» de desarrollo y estudiar las implicaciones que tiene para su propia enseñanza. Entonces se les proporciona un plan de estudios formal para que utilicen con los estudiantes en el transcurso del año académico. El plan de estudios ofrece a profesores y estudiantes un lenguaje común y un marco conceptual desde el cual se intentan resolver cuestiones como por qué en el pasado algún alumno no ha podido con las matemáticas. El profesor inculca en el niño que el aprendizaje está en función del esfuerzo y no de una capacidad innata. El currículo ayuda a los estudiantes a incrementar su concienciación, para que así puedan afirmar por sí mismos sus necesidades de auto-desarrollo. Una afirmación de este tipo por parte de los alumnos es un prerrequisito crítico para enfrentarse a los obstáculos en el camino de su desarrollo, y para la adquisición de actitudes y hábitos que aseguren el éxito en muchos proyectos, incluyendo en el programa de álgebra.

En 1988, una profesora de sexto del Programa Abierto comenzó a dar el programa de estudios "Efficacy" a todos los alumnos de sexto dos veces por semana. Nos comenta:

"Todos nos consideramos buenos profesores, pero aun así sabemos que estamos fallando a algunos alumnos. Bob nos habló de un método que podía ayudarnos a ayudar a estos alumnos a tener un rendimiento superior. Nos dimos cuenta de lo que eso significaría, no sólo para estos estudiantes sino para todos los niños de la clase, y a partir de ahí lo que significaría para la comunidad en general."

\section{El Proyecto continúa}

El Proyecto Algebra continúa como parte del Programa Abierto. El programa de estudios "Efficacy» y el de Algebra se da a los estudiantes de sexto, y todo séptimo y octavo da clases de álgebra. Ahora el proyecto está desafiando a otros colegios para que tomen la decisión política de cambiar su programa de estudios de matemáticas. Por ejemplo, se están celebrando reuniones con administradores y profesores del área de Boston donde hay tres colegios que se han of recido voluntarios para experimentar con los programas de estudios de los Proyectos Efficacy y Algebra, y recibir entrenamiento para su puesta en marcha. Moses también ha comenzado a preparar a un grupo de profesores de Atlanta de la etapa intermedia escolar. Actualmente, el Proyecto está explorando relaciones con otros sistemas escolares en otras ciudades. 


\section{Conclusiones}

\section{Organización de la comunidad e innova- ciones educativas}

La organización de la comunidad como enfoque para las innovaciones educativas difiere de las intervenciones educarivas tradicionales de diversas formas importantes. El principio de "posa tu cubo donde estés" contrasta con los programas de investigación que tienen su origen en las universidades, donde estudiosos diseñan programas de intervención que hipotetizan producirán resultados que articulan por adelantado y que son replicables. Los investigadores en las universidades y. en las empresas asesoras deben elaborar intervenciones que estén bien diseñadas y altamente articuladas, con el fin de convencer a las entidades de financiación que sus proyectos son prometedores. Según el enfoque de la investigación, el investigador generalmente selecciona como objetivos a barrios, colegios u organizaciones concretas, en base a factores demográficos u otras características igualmente cuantificables. Además, los investigadores están intelectualmente anclados en su propia disciplina y observan los problemas a través de lentes que están en consonancia con sus disciplinas, en vez de hacerlo a través de los ojos de la comunidad.

En contraste con el investigador con base en la universidad, el organizador que trabaja siguiendo la tradición de Ella es gradualmente reconocido por los miembros de la comunidad como alguien comprometido a luchar por su bienestar global. El organizador se sumerge en la vida de la comunidad, aprendiendo sus puntos fuertes, recursos, preocupaciones y formas de llevar los asuntos. El organizadór no posee un plan global y detallado con el que remediar un problema, sino que adopta una visión «evolutiva» de su papel en la construcción de una solución. Comprende que las preocupaciones cotidianas de la comunidad pueden transformarse en preguntas políticas más amplias de importancia general. La forma que adoptarán no siempre se conoce de antemano.

Una vez que se han identificado las preguntas políticas, la agenda del organizador debe mantenerse simultáneamente centrada y fluida - nítidamente centrada en los objetivos a largo plazo, pero fluida con respecto a cómo se alcanzará el objetivo-. El organizador averigua el punto de vista de aquellos niembros de la comunidad que poseen fuertes intereses en el asunto, y de una manera informal educa a los miembros de la comunidad que no están implicados pero cuyos intereses están en juego. La tarea del organizador es ayudar a los miem- bros de la comunidad a expresar sus opiniones, hacerse preguntas entre sí y luego construir un consenso, un proceso que generalmente lleva bastante tiempo.

Mejorar el programa de matemáticas y el proceso curricular en la etapa intermedia educariva de un centro escolar se ha convertido gradualmente en el foco del Proyecto Algebra. Al principio Moses no sabía que: el proyecto se convertiría en un vehículo para plantear cuestiones como el sistema de agrupamiento de los estudiantes según su capacidad, la enseñanza efectiva para estudiantes. de color, o el papel de la comunidad en la toma de decisiones educativas. No podía imaginarse que se pondría en marcha un interés por la enseñanza de álgebra, más allá de la clase de su hija, dirigido a estudiantes en la etapa intermedia escolar de áreas urbanas.

Como hemos visto, las innovaciones del Programa contaban con la participación de toda la comunidad: profesores, padres, administradores escolares, tutores, y consultores de la comunidad de Greater Boston. En su revisión de los programas que han contribuido a romper el ciclo de desventaja, Lisbeth Schorr (1988) acentúa la importancia de adoptar enfoques hacia las reformas que sean amplios, flexibles e intensivos ${ }^{\text {? }}$ :

"La razón por la que muchas intervenciones no han sido efectivas no es porque no se pueda ayudar a las familias seriamente desfavorecidas, sino porque hemos intentado atacar problemas complejos, fuertemente arraigados, con fragmentos aislados de ayuda; con ayuda ofrecida a regañadientes en incursiones rápidas sin ningún seguimiento; con ayuda diseñada no tanto para cubrir las necesidades de los beneficiarios como para ajustarse a la conveniencia profesional o burocrática; ayuida que puede servir a familias de clase media pero que con frecuencia es irrelevante para las familias que están luchando por sobrevivir"(pp. 263-264)

El trabajo de encontrar soluciones nuevas, crear una amplia base de apoyo y superar barreras lleva tiempo. El esfuerzo realizado por Moses de trabajar con profesores, padres y administradores para transformar el currículo de matemáticas y los procesos curriculares que se dan en la etapa intermedia escolar dentro del Programa Abierto comenzó hace siete años. Pero tomamos nota que los esfuerzos de James Comer por lograr una reforma extensa en dos colegios de New Haven tardaron quince años en producir mejoras notables en las puntuaciones de los estudiantes en las pruebas de rendimiento administradas (Comer, 1988; $1980)^{13}$. Las reformas duraderas son posibles, pero no existen atajos cuando su aplicación comienza desde la base. 
En el Programa Abierto, el profesorado se ofreció voluntariamente a participar, comprometiéndose a trabajar en equipo para descubrir mejores formas de enseñar matemáticas y luchando por llegar a un acuerdo. Los padres participaban activamente como estudiantes, dando apoyo, colaborando y tomando decisiones. Los estudiantes establecieron voluntariamente sus propias metas, y cuatro veces por semana llegaban a las 7,30 de la mañana para asistir a las aulas de estudio. Los administradores escolares dieron su apoyo al profesorado mientras éstos probaban nuevas estrategias, trabajaron para asegurar una financiación, y actuaron como portavoces del Proyecto. Se reconocieron los puntos fuertes de diversos contribuidores y se les autorizó a que adaptasen, crearan y evaluaran el progreso logrado en alcanzar una visión compartida.

Otros autores han aprendido que el compromiso hacia el cambio se da realmente a través del esfuerzo por resolver un problema y de modelar las soluciones. Schorr (1988) nos informa:

«El Dr. Comer quería asegurarse de que comprendía que la esencia de su intervención es un proceso, no un paquete de materiales, métodos educativos o técnicas. "Es la creación de una sensación de comunidad y de dirección para los padres, el personal del centro y los estudiantes por igual" " (p. 234)

Comer está apuntando hacia el hecho de que las innovaciones significativas deben transformar la cultura, y la transformación exige una amplia base de apoyo voluntario. Es crucial que los participantes tengan tiempo para comprender una idea, explorar su compromiso personal y adaptar las innovaciones a sus necesidades.

Henry Levin (1988) también subraya la importancia del proceso $^{14}$. Manifiesta que:

"Hay dos suposiciones principales que subyacen al enfoque organizativo: Primero, la estrategia debe "delegar autoridad" en todos los participantes principales y elevar su sencido de eficacia y de responsabilidad hacia los resultados para el colegio. Segundo, el enfoque debe construirse sobre la enorme cantidad de puntos fuertes de los participantes en vez de criticar sus debilidades". (p. 5)

A muchos les resultará de utilidad seguir el precepto "posa tu cubo donde estés", como lo hizo James Escalante en Los Angeles, cuando comenzó a dar cálculo a jóvenes desfavorecidos. El punto de partida para la reforma es menos importante que el hecho de que la cuestión resulte lo suficientemente poderosa e inspiradora como para generar entusiamo, sacar a la luz cuestiones políticas más amplias, imponer un liderazgo dedicado y servir de vehículo para la participación de la comunidad.

\section{La financiación para apoyar las innovaciones}

El Proyecto Algebra no se hubiera desarrollado de la forma en que lo hizo de no haber sido por la beca de McArthur que, concedida sin ninguna condición restrictiva, permitió a Moses trabajar en el Programa Abierto durante cinco años sin tener que dar cuenta de la forma en que invertía su tiempo. La obtención de más financiación ha resultado difícil. Durante dieciocho meses, el Wheelock College en Boston financió a Moses mientras éste buscaba recursos económicos para poder conceder tiempo libre al personal docente, cubrir el coste de materiales y de reproducciones, y asegurar la posibilidad de consultar a la comunidad académica. Moses sigue dedicando una enorme cantidad de su tiempo a lograr obtener financiación a largo plazo para mantener la continuación y difusión del Proyecto Algebra.

Encontrar apoyo financiero puede convertirse en una lucha agotadora para muchos esfuerzos innovadores. Las fuentes de financiación nacional vacilan a la hora de financiar proyectos con un liderazgo de base, un enfoque a largo plazo, centrado en la comunidad, y una filosofía que encuadra las cuestiones educativas en términos técnicos, pero también en términos políticos. La reducción de los presupuestos estatales y locales también amenaza el compromiso con las reformas globales y a largo plazo. Pero solamente cuando se abordan cuestiones políticas de importancia (por ejemplo, que todos los niños pueden beneficiarse del álgebra y deberían tener acceso a ella durante los años de la etapa intermedia escolar) podemos descubrir las formas más adecuadas para organizar el conocimiento, desarrollar el programa de estudios y fomentar la participación de la familia, el colegio y la comunidad.

\section{Transformando la cultura escolar}

Los profesores y los padres que tomaron parte en el Programa Abierto llegaron a creer que el sistema de agrupar a los estudiantes según su capacidad, reducía seriamente la capacidad de los estudiantes de color de la etapa intermedia escolar para aprender tanto como realmente podían. Cuestionar esta política de grupos fue el primer paso hacia un cambio global. Otros autores coinciden que al hacer diferencias entre los estudiantes se perjudica a los más desfavorecidos o a los que entran en 
los niveles inferiores de rendimiento ${ }^{15}$. Tras articular una visión de altas expectativas en álgebra para todos los estudiantes, los participantes trabajaron para cambiar la cultura escolar, con el fin de que la política escolar, las estrategias de enseñanza y el programa "Efficacy” de estudios pudieran en conjunto ayudar a los estudiantes.

El proyecto trata de la importancia de la familia como eslabón para el éxito escolar. Henderson (1987) concluye su revisión de las investigaciones sobre la participación de los padres en el rendimiento de los estudiantes, afirmando categóricamente que "los datos son indiscutibles: la participación de los padres mejora el rendimiento del estudiante» (p. 1). Este resultado es aplicable a familias tanto de nivel económico medio como bajo, a estudiantes en diferentes niveles de escolaridad y en una amplia gama de intervenciones. Al darse, cada vez más, una mayor diversidad en la población de los Estados Unidos, es absolutamente fundamental que los colegios y las familias se unan para definir y apoyar el éxito escolar. La continuidad entre el hogar y la escuela debe forjarse para todos los niños, y debemos apoyarnos en los puntos fuertes y los recursos que las familias pueden aportar.

\section{El programa de estudios y el proceso curricular}

Uno de los puntos fuertes del profesorado y el personal voluntario en el Programa Abierto era su curiosidad sobre por qué algunos niños no tenían éxito en matemáticas, y su disposición a explorar la posibilidad de que uno de los factores responsables podría ser sus estrategias de enseñanza. Moses y el resto del profesorado se convirtieron en investigadores de los procesos que se daban en el aula; analizando los errores de los estudiantes, localizando nudos conceptuales y experimentando con materiales y procesos de enseñanza que pudieran mejorar el desarrollo matemático de los estudiantes. Un programa de estudios de transición en sexto curso, que permite a los estudiantes relacionar las experiencias cotidianas con los conceptos matemáticos representados simbólicamente, debería ser ampliamente difundido.

En 1964 la atención nacional se centró en los ciudadanos del sur privados de sus derechos civiles. En 1989 existe otro tipo de privación de derechos, al haber tantos niños de color pobres, tanto nativos como emigrantes, a quienes se les niega el acceso a programas educativos y a una enseñanza que se basa en el éxito escolar. El éxito del Proyecto Algebra queda como reto para los profesores, administradores e investigadores de los colegios públicos, y más importante aún para aquellos individuos que tradicionalmente han abogado por una democratización de la sociedad y las escuelas: ¿Emprenderéis una campaña a favor de la destreza matemática que asuma que todos los estudiantes de la etapa intermedia escolar pueden y deben aprender álgebra mientras simultáneamente se delega autoridad en la comunidad y en la familia del niño? ¿Organizaréis en el espíritu de Ella?

\section{Notas}

1 Fundi es un término Swahili que se da a una persona experta en un campo valorado por la sociedad y que transmite su arte a los jóvenes a través de cjemplos y de instrucción. Ella Baker fue una fur.di para los niembros del SNCC enseñándoles el arte de organización de la comunidad.

- Un lugar tranquilo de este tipo era el Condado de Amite, situado en una parte remota del sudoeste de Mississippi, donde en el verano de 1961 la familia de E. W. Steptoe dio la bienvenida a la comunidad a Bob Moses, el primer secretario de campo del SNCC. El Sr. Steptoe era el presidente de la rama del NAACP del Condado de Anite a finales de los años 50, cuando el sheriff del condado realizó una redada durante una reunión y confiscó los libros del grupo, exponiendo de esta forma a los niembros a represalias económicas y peligro físico. Cuando la primera oleada de organizadores del SNCC comenzó a extenderse por el área rural del Sur, en lugares como la granja de los Steptoe toda actividad se había paralizado por completo.

${ }^{3}$ La expresión "cast down your bucket where you are" fue utilizada por Booker $\mathrm{T}$. Washington en su discurso en la Exposición de Arlanta el 18 de septienbre de 1895.

${ }^{+}$El Colegio King es un edificio grande y moderno construido en el solar de un colegio que había servido a la comunidad negra de Cambridge durante muchos años. A finales de los años 70 , el Colegio King tenía en marcha cuatro programas dirigidos a los cursos desde preescolar a octavo: un programa regular llevado por personal del colegio anterior; un Programa Abierto $\mathrm{y}$ dos programas más pequeños, uno bilingüe $\mathrm{y}$ otro de necesidades especiales.

5 Debido a que algunos autores de este artículo, aunque no todos, son también el objeto de discusión, hemos decidido hacer las referencias en tercera persona con el fin de evitar confusiones.

- El cuarto estudiante optó por ir a una escuela privada de secundaria y no se presentó al examen.

${ }^{7}$ Véase Delpit (1986) para una discusión de las diferencias entre las necesidades educativas de los niños de las clases mayoritarias y las de los niños de las minorías.

${ }^{8}$ Véase Fordham (1988) para una discusión sobre las tensiones que padecen los negros de alto rendimiento cuando se esfuerzan por alcanzar el éxito académico. 
Y Sólo en Mississippi, donde la totalidad del estado estaba estructurado de acuerdo con una tradición de organización de la comunidad, y donde la cuestión del derecho al voto se percibió como una pregunta política más amplia.

${ }^{10}$ Este modelo es una síntesis de ideas tomadas de tres fuentes. La primera fue el propio Programa Abierto. Moses observó la práctica docente en el Programa Abierto y asistió a talleres con profesores en donde Virginia Chalmers y otros explicaron las ideas de enseñanza y aprendizaje que habían desarrollado para los cursos de primaria. El segundo fue la noción de Quine (1981) de "matematización in situ». "Un progresivo refinamiento y reglamentación de expresiones normales: esto es lo que llevó a la aritmética, la lógica simbólica y la teoría fija, y esto es la matematización" (p. 150). Quine insistió en que "la teoría fija, la aritmética, $y$ la lógica simbólica son todos ellos productos de la matematización directa del discurso interpretado normalmente..." (p. 151). La tercera fuente fue Dubinsky (1987), quien compartió su visión de que en el futuro la educación matemática estaría centrada en un «proceso curricular fijo" en vez de en un "curriculo fijo".

1 El modelo Efficacy de desarrollo intelectual se basa en la motivación. El papel de la motivación en el autodesarrollo fue estudiado por Jeffrey Howard, Director del Instituto Efficacy, quien en colaboración con educadores desarroltaron el modelo resumido aquí.

12 Corno participante en el Grupo de Trabajo de la Universidad de Harvard sobre la Vida Temprana y la Adolescencia, Lisbeth Schorr consideraba que con los conocimientos actualmente existentes, la sociedad podía evitar los resultados perjudiciales asociados con un medio desfavorecido para los adolescentes, como, por ejemplo, el embarazo en la adolescencia, el crimen juvenil, el fracaso escolar y el desempleo. Schorr visitó distintos programas de salud y programas educativos que habían logrado romper el ćrculo vicioso de la desventaja, y descubrió que todos ellos compartían un enfoque amplio, flexible e intensivo hacia la reforma.

${ }^{13}$ En 1968 James Comer, un psiquiatra del Centro de Estudios de la Infancia de Yale, comenzó un programa de reforma en dos colegios de New Haven que tenían las puntuaciones en rendimiento más bajas y el peor historial de asistencia y conducta del sistema. Hoy en día, aunque la comunidad sigue siendo pobre, estos colegios pueden presumir de tener las puntuaciones en rendimiento más altas dentro del sistema de New Haven (tercero y cuarto), no tienen problemas serios de conducta y poseen un historial de asistencia muy alto. Los componentes esenciales de la reforma, ahora extendidos por otros catorce centros, incluyen un Equipo Escolar de Dirección y Planificación (formado por el director, los padres y el profesorado), un equipo de salud mental, que proporciona un servicio coordinado a niños en conflicto, y una amplia participación de los padres.

${ }^{14}$ Henry Levin es el director de un proyecto de gran éxito en el Area de la Bahía de San Francisco denominado Proyecto Escolar Acelerado, cuya misión es "introducir a los niños en la vía principal educativa" para que puedan beneficiarse al máximo de la escolarización futura y de sus oportunidades como adultos" (1988, p. 3).

15 Levin (1988) arguméntó que la principal razón del fracaso de muchos niños desfavorecidos es la baja expectativa del profesor, que a su vez lleva a programas de recuperación basados en planes de estudios de repetición y repaso que resultan muy aburridos. Peterson (1989) realizó un estudio en Utah en el que concluyó que el agrupamiento de estudiantes de acuerdo con su capacidad es dañino para los estudiantes de recuperación, y que la participación en programas acelerados es una ruta más efectiva para alcanzar un rendimiento superior.

\section{Referencias}

Comkr, J. (1988): Maggie's American dream. Nueva York. New American Library.

Combr, J. (1980): School power. Nueva York: Free Press.

Di:LPT, L. (1986): Skills and other dilemmas of a progressive Black educator. Harvard Educational Review, 56, 379-385.

Dubinsky, E. (1987). How Piaget's and related work should influence $K-12$ curriculum design. Manuscrito no publicado.

FordHAM, S. (1988). Racelessness as a factor in Black sudents' school success: Pragmatic strategy or Pyrrhic victory? Harvard Educational Review, 58, 54-84.

Henderson, A. (1987). The evidence continues to grow: Parent involvement improves student acbievement. Columbia, MD: National Committee for Citizens in Education.

Llivin, H. (1988). Don't remediate: Accelerate. En Proceedings of the Stanford University Centennial Conference, Accelerating the Education of At-Risk Students. (Disponible en: Center for Educational Research, CERAS Building, 402-South, Stanford University, Stanfor, CA 94305.)

National. Science Foundation. (1989). Materials for middle school mathematics instruction. Catalog of Federal Domestic Assistance No. 47.067, Materials Development, Research, and Informal Science Education.

PrTtikson, J. (1989). Remediation is no remedy. Educational Leadership, 46(6), 24-25.

Quine, W. V. (1981). Theories and things. Cambridge: Harvard University Press.

Saxon, J. H., Jr. (1982). Algebra I: An incremental development. Norman, OK: Saxon Publishers.

Schork, L. (1988). Within our reach: Breaking the cycle of disadvantage. Nueva York: Anchor Press/Doubleday.

Usiskin, Z. (1987). Why elementary algebra can, should, and must be an eighth-grade course for average students. Mathermatics Teacher, 80, 428-437 


\section{El Proyecto Algebra: Un movimiento organizativo inspirado en Ella. R. P. Moses, M. Kamii, S. McAllister y J. Howard. CLEE, 1991, 9, pp. 3-17}

Agradecimientos: Los autores desean agradecer a las siguientes personas su contribución a este artículo: Theresa Perry, Daniel Cheever, Barney Brawer, Ceasar McDowell y, finalmente, al profesorado y administradores del Programa Abierto del Colegio Martín Lutero King, Jr.

Dirección de los autores: Susan McAllister Swap, Wheelock College, Boston. Jeffrey Howard, Efficacy Institute, Lexington, Massachusetts.

Artículo original: The Algebra Project: Organizing in the Spirit of Ella. Harvard Educational Review, 1989, 54(4), 423-443. Reproducido con autorización de los autores $\mathrm{y}$ de Harvard Educational Review. Traducción de Paloma Linares $\odot$ de la traducción al castellano, CL\&E, 1991

( ) de todos los artículos. Deberá solicitarse por escrito autorización de CL\&E para el uso en forma de facsímil, fotocopia o cualquier otro medio de reproducción. CL\&E se reserva el derecho de interponer las acciones legales necesarias en aquellos casos en que se contravenga la ley de derechos de autor. 